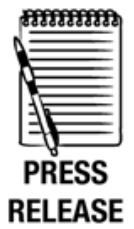

- Additional tables are published online only. To view these files please visit the journal online (http://bjo.bmj. com/content/96/5.toc).

1 Division of Population Health Sciences and Education, St George's, University of London, London, UK

${ }^{2}$ London School of Hygiene \& Tropical Medicine, London, UK ${ }^{3}$ Moorfields Eye Hospital, London, UK

\section{Correspondence to}

Dr Christopher G Owen, Division of Population Health Sciences and Education, St George's, University of London, Cranmer Terrace, London SW17 ORE, UK; cowen@sgul.ac.uk

Accepted 28 December 2011

Published Online First

13 February 2012

\section{(2) UNLOCKA}

This paper is freely available online under the BMJ Journals unlocked scheme, see http:// bjo.bmi.com/site/about/ unlocked.xhtml

\title{
The estimated prevalence and incidence of late stage age related macular degeneration in the UK
}

\author{
Christopher G Owen, ${ }^{1}$ Zakariya Jarrar, ${ }^{1}$ Richard Wormald, ${ }^{2,3}$ Derek G Cook, \\ Astrid E Fletcher, ${ }^{2}$ Alicja R Rudnicka ${ }^{1}$
}

\section{ABSTRACT \\ Background UK estimates of age related macular degeneration (AMD) occurrence vary.}

Aims To estimate prevalence, number and incidence of AMD by type in the UK population aged $\geq 50$ years

Methods Age-specific prevalence rates of AMD obtained from a Bayesian meta-analysis of AMD prevalence were applied to UK 2007-2009 population data. Incidence was estimated from modelled age-specific prevalence.

Results Overall prevalence of late AMD was 2.4\% (95\% credible interval (Crl) $1.7 \%$ to $3.3 \%$ ), equivalent to 513000 cases (95\% Crl 363000 to 699000$)$; estimated to increase to 679000 cases by 2020 . Prevalences were $4.8 \%$ aged $\geq 65$ years, $12.2 \%$ aged $\geq 80$ years.

Geographical atrophy (GA) prevalence rates were 1.3\% (95\% Crl $0.9 \%$ to $1.9 \%$ ), $2.6 \%$ (95\% Crl $1.8 \%$ to $3.7 \%$ ) and $6.7 \%(95 \% \mathrm{Crl} 4.6 \%$ to $9.6 \%)$; neovascular $\mathrm{AMD}$ (NVAMD) $1.2 \%$ (95\% Crl $0.9 \%$ to $1.7 \%$ ), $2.5 \%$ (95\% Crl $1.8 \%$ to $3.4 \%)$ and $6.3 \%(95 \% \mathrm{Crl} 4.5 \%$ to $8.6 \%)$, respectively. The estimated number of prevalent cases of late AMD were $60 \%$ higher in women versus men (314000 cases in women, 192000 men). Annual incidence of late AMD, GA and NVAMD per 1000 women was 4.1 (95\% Crl 2.4\% to 6.8\%), 2.4 (95\% Crl $1.5 \%$ to $3.9 \%$ ) and $2.3(95 \% \mathrm{Crl} 1.4 \%$ to $4.0 \%)$; in men $2.6(95 \% \mathrm{Crl} 1.5 \%$ to $4.4 \%), 1.7(95 \% \mathrm{Crl} 1.0 \%$ to $2.8 \%)$ and $1.4(95 \% \mathrm{Crl} 0.8 \%$ to $2.4 \%)$, respectively. 71000 new cases of late AMD were estimated per year. Conclusions These estimates will guide health and social service provision for those with late AMD and enable estimation of the cost of introducing new treatments.

\section{INTRODUCTION}

Age related macular degeneration (AMD) is a major cause of ocular morbidity in high income countries, accounting for over half of blind and partial sight certifications in the UK. ${ }^{1}$ However, the number registered underestimates the number with visual loss caused by the condition, as it excludes those who choose not to be registered. Moreover, this figure does not include the appreciable number of those with the condition who do not qualify for registration. There have been a number of attempts to quantify the number with $\mathrm{AMD}$ in the $\mathrm{UK}^{2}{ }^{3}$ but these estimates differ, and are heavily dependent on the characteristics of studies used to generate the figures; in particular, age of the study sample, case definition of $\mathrm{AMD}$, and whether fundus imaging and independent grading have been used. We have previously quantified the magnitude of effect these characteristics have on the prevalence of AMD. ${ }^{4}$
While a treatment for geographical atrophy (GA) remains to be established, treatments for neovascular AMD (NVAMD) using photodynamic therapy and antivascular endothelial growth factors exist; the latter is being increasingly used to limit progression of the condition. ${ }^{5}$ In order to plan adequate healthcare provision, estimates of incidence are required, but these differ between studies. $^{6-9}$ Estimates suggest that the number eligible for treatment has increased fivefold over a relatively short period. ${ }^{10-12}$ These estimates are based on certification data ${ }^{10}$ or from a small number of population cohorts, which have followed up individuals from 5 to 15 years. ${ }^{8} 1314$ Extrapolating from these studies to the population at large is highly sensitive to the age distribution of the study population and duration of follow-up. A more robust approach is to use age-specific prevalence rates of late AMD. Data modelled from many studies allow a wider range of ages to be represented compared with the limits of a single incidence study. Robust estimates of prevalence at older ages are of particular importance as this is where late $\mathrm{AMD}$ is most prevalent. We have therefore applied age-specific prevalence rates from our recent review (modelled using Bayesian metaanalysis over a wide range of ages) ${ }^{4}$ to UK population data to provide estimates of the number of prevalent cases and incidence of late $\mathrm{AMD}, \mathrm{GA}$ and NVAMD.

\section{MATERIALS AND METHODS Age-specific prevalence data}

We estimated the age-specific prevalence of late $\mathrm{AMD}, \mathrm{GA}$ and NVAMD (for men, women and genders combined) using a Bayesian meta-analysis of 31 populations studies with a combined population of 57173 (with 1571, 455 and 464 cases of late $\mathrm{AMD}, \mathrm{GA}$ and NVAMD cases, respectively). ${ }^{4}$ These prevalence estimates represent the most complete meta-analysis of AMD prevalence in white populations (from Europe, North America and Australia) largely similar to the middle-aged and older population of the UK. Prevalence estimates allowed for study characteristics, such as age of the sample, examination methods and definitions of disease (internationally recognised definitions being preferred). ${ }^{15} 16$ Prevalence estimates from the Bayesian model were produced by year from 50 to 97 years of age (the oldest reported age in the studies included in the meta-analysis) for men, women and genders combined (Web table 1) based on 'either eye' case definitions (including at least one eye, worst eye, one or both eyes) using the International Classification System or Wisconsin Age- 
Related Maculopathy Grading System and fundus photography. ${ }^{15} 16$ A 95\% credible interval (CrI) of prevalence by year was also obtained from the Bayesian model: a 95\% CrI represents the range of values within which the true prevalence is expected to lie with $95 \%$ probability.

\section{UK population characteristics}

Demographic characteristics for the population of the UK were obtained from the Office of National Statistics. The mean yearly population from 50 to 97 years of age was obtained from estimates for the years 2007-2009. ${ }^{17-19}$ Prevalence estimates for each year of age from the Bayesian model were applied to these population figures. The Office of National Statistics also provides estimates of the probability of dying at each year of age $^{20}$ as well as projections of UK population numbers over time. ${ }^{21}$ Population projections up to 2020 were used as data beyond this may be less reliable.

\section{Estimating incidence from prevalence}

Estimating incidence from age-specific prevalence data has been carried out previously for open angle glaucoma. ${ }^{22}$ We used this method to estimate the annual incidence by age of late $\mathrm{AMD}$, GA and NVAMD in men and women between the ages of 50 and 97 years. Prevalence estimates for each year of age were used to determine the probability of AMD per year of age. The probability is then converted into an incidence assuming that: (i) the outcome of interest is life-long after diagnosis; (ii) mortality risk is the same in those with and without the disease; and (iii) the
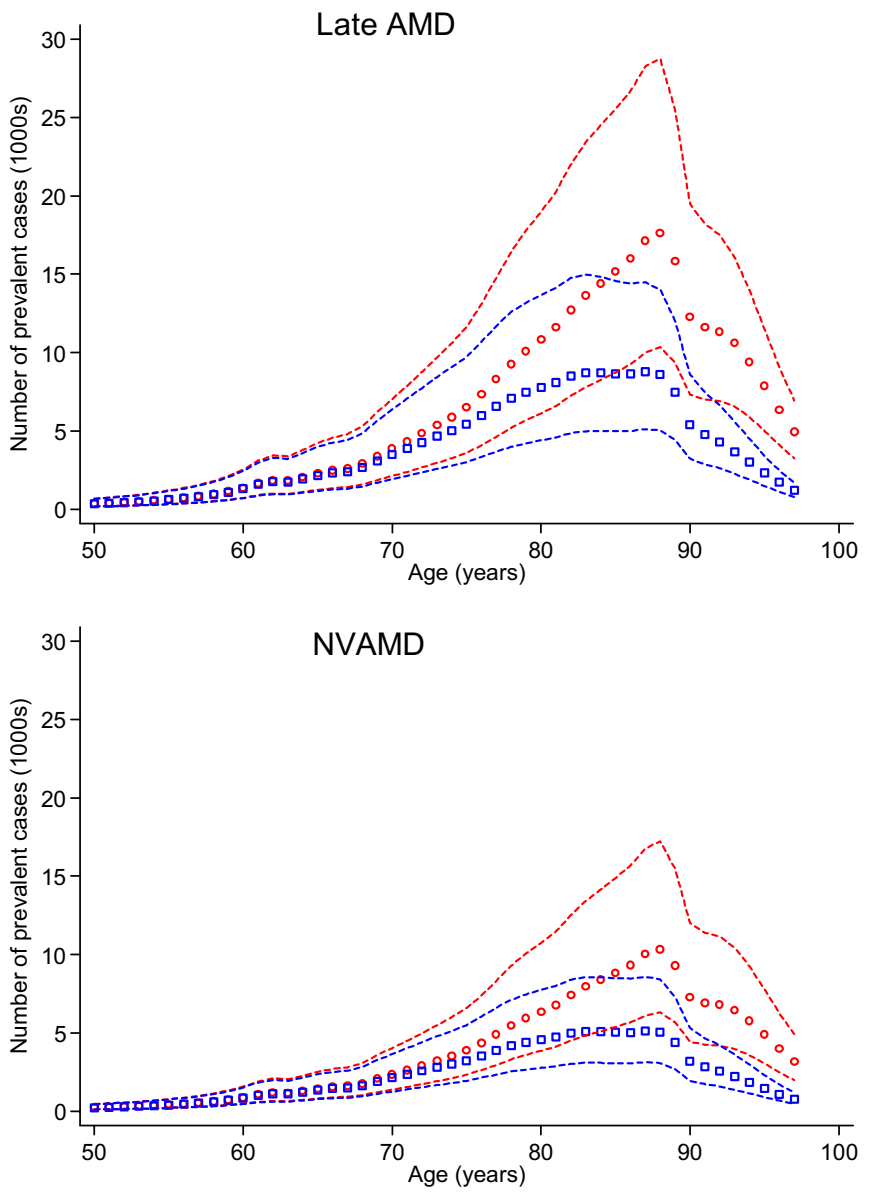

disease is stable in the population (with risk factors for the condition remaining constant).

\section{RESULTS}

The prevalence of late $\mathrm{AMD}$ standardised to the UK population aged 50 years or more was $2.4 \%$ (95\% CrI $1.7 \%$ to $3.3 \%$ ), increasing to $4.8 \%$ (95\% CrI 3.4\% to $6.6 \%$ ) in those aged 65 years or more and $12.2 \%$ (95\% CrI $8.8 \%$ to $16.3 \%$ ) in those aged 80 years or more. Prevalences for GA were 1.3\% (95\% CrI $0.9 \%$ to $1.9 \%), 2.6 \%$ (95\% CrI $1.8 \%$ to $3.7 \%$ ) and $6.7 \%(95 \% \mathrm{CrI}$ $4.6 \%$ to $9.6 \%$ ) and for NVAMD $1.2 \%(95 \% \operatorname{CrI} 0.9 \%$ to $1.7 \%)$, $2.5 \%$ (95\% CrI $1.8 \%$ to $3.4 \%$ ) and $6.3 \%$ (95\% CrI $4.5 \%$ to $8.6 \%)$, respectively. These estimates applied to the UK population result in approximately 513000 prevalent cases of late AMD (95\% Cr I 363000 to 699000), 276000 cases of GA (95\% CrI 188000 to 396000$)$ and 263000 cases of NVAMD (95\% CrI 185000 to 361000$)$. UK age-specific prevalence rates of late $\mathrm{AMD}, \mathrm{GA}$ and NVAMD are shown for different age groups in Web table 1 for men and women separately; the number of cases is shown graphically in figure 1. The Bayesian model showed little gender difference in GA prevalence, but slightly higher prevalence in women for late $\mathrm{AMD}$, which was explained by the higher prevalence of NVAMD in women. ${ }^{4}$ Application of these prevalence rates to the UK population, in combination with larger numbers of older women at older ages, results in marked gender differences in the number of prevalent cases, especially between 80 and 90 years of age (figure 1). Overall, the estimated number of prevalent cases of late $\mathrm{AMD}$ was $60 \%$ higher in

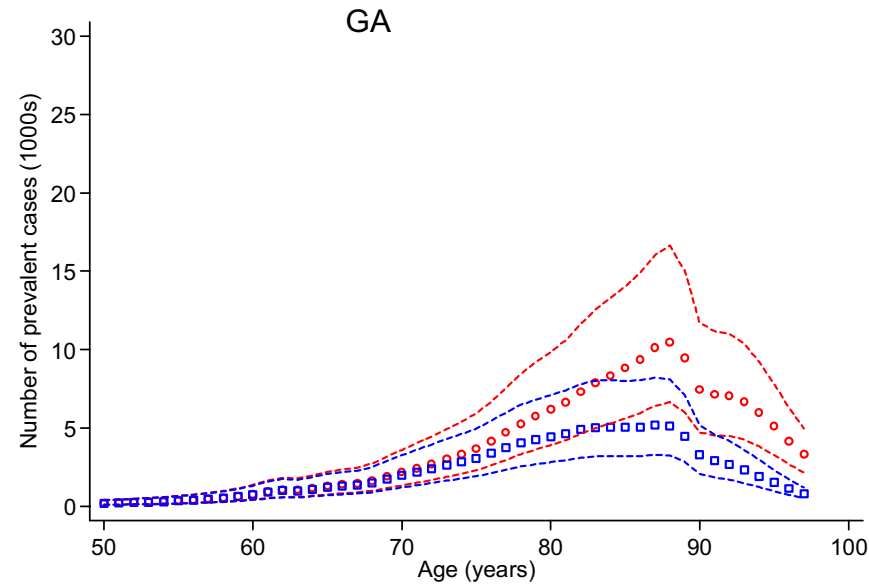

Figure 1 Number of prevalent cases (in thousands) of late age related macular degeneration (AMD), geographical atrophy (GA) and neovascular AMD (NVAMD) in men (open blue squares) and women (open red) by 1-year age groups. The dashed blue and red lines are the $95 \%$ credible intervals for men and women, respectively. 
women compared with men, with 314000 cases in women and 192000 in men. Beyond 90 years of age, there is a sharp decline in the number of AMD cases in both genders (figure 1). Application of these prevalence rates to population projections suggests a linear increase in the number of AMD cases by 2020. There are estimated to be 394000 women and 285000 men (679000 in all) with late AMD by 2020 (figure 2), which is an increase of a third. Data for GA and NVAMD show similar increases (data available from the authors).

The overall number of incident cases in those aged over 50 years for late AMD, GA and NVAMD in women was $4.1(95 \%$ CrI 2.4 to 6.8), 2.4 (95\% CrI 1.5 to 3.9$)$ and 2.3 (95\% CrI 1.4 to $4.0)$ in the UK per 1000 per year; in men it is 2.6 (95\% CrI 1.5 to 4.4), 1.7 (95\% CrI 1.0 to 2.8) and 1.4 (95\% CrI 0.8 to 2.4), respectively. Incidence by age (figure 3 , Web table 2 ) shows higher rates of NVAMD in women, and slightly higher rates in women for late $\mathrm{AMD}$. Application of these incidence rates to the UK population results in higher numbers of new late AMD, GA and NVAMD cases per year in women compared with men, being most marked between 80 and 90 years of age, with a sharp decline thereafter. The total number of new cases of late AMD, GA and NVAMD per year in men and women was 25344 (95\% CrI 14500 to 43900), 16700 (95\% CrI 10200 to 27200) and 13400 (95\% CrI 7900 to 23200$)$ and 45900 (95\% CrI 26600 to $77000), 27000$ (95\% CrI 16800 to 44000$)$ and 26400 (95\% CrI 15800 to 45100$)$, respectively.

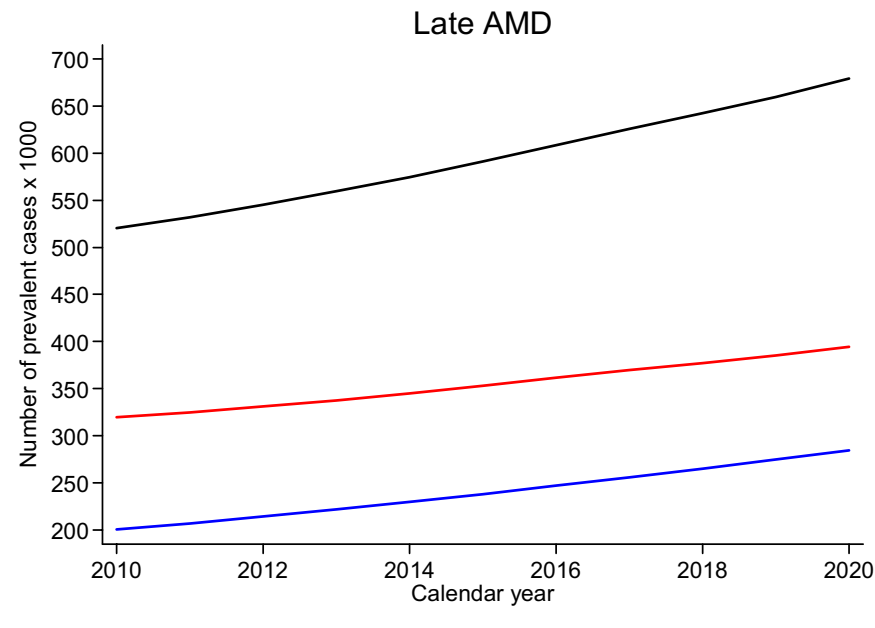

Figure 2 Projected number of prevalent cases (in thousands) of late age related macular degeneration (AMD) in men (blue line) and women (red line) and combined (black line) from 2010 to 2020.

\section{DISCUSSION}

We have applied findings from a recent systematic review ${ }^{4}$ based on 31 populations of European ancestry with a wide age range (50-97 years) (including 18 populations from Western Europe with four from the $\mathrm{UK}^{23-26}$ ) to the population of the UK to
Figure 3 Graphs on the left had side represent the annual incidence (number per 1000) of late age related macular degeneration (AMD), geographical atrophy (GA) and neovascular AMD (NVAMD) in men (open blue squares) and women (open red circles). The dashed blue and red lines are the $95 \%$ credible intervals for men and women, respectively. Graphs on the right hand side represent number of new cases per year in men (blue line) and women (red line) as applied to the UK population.
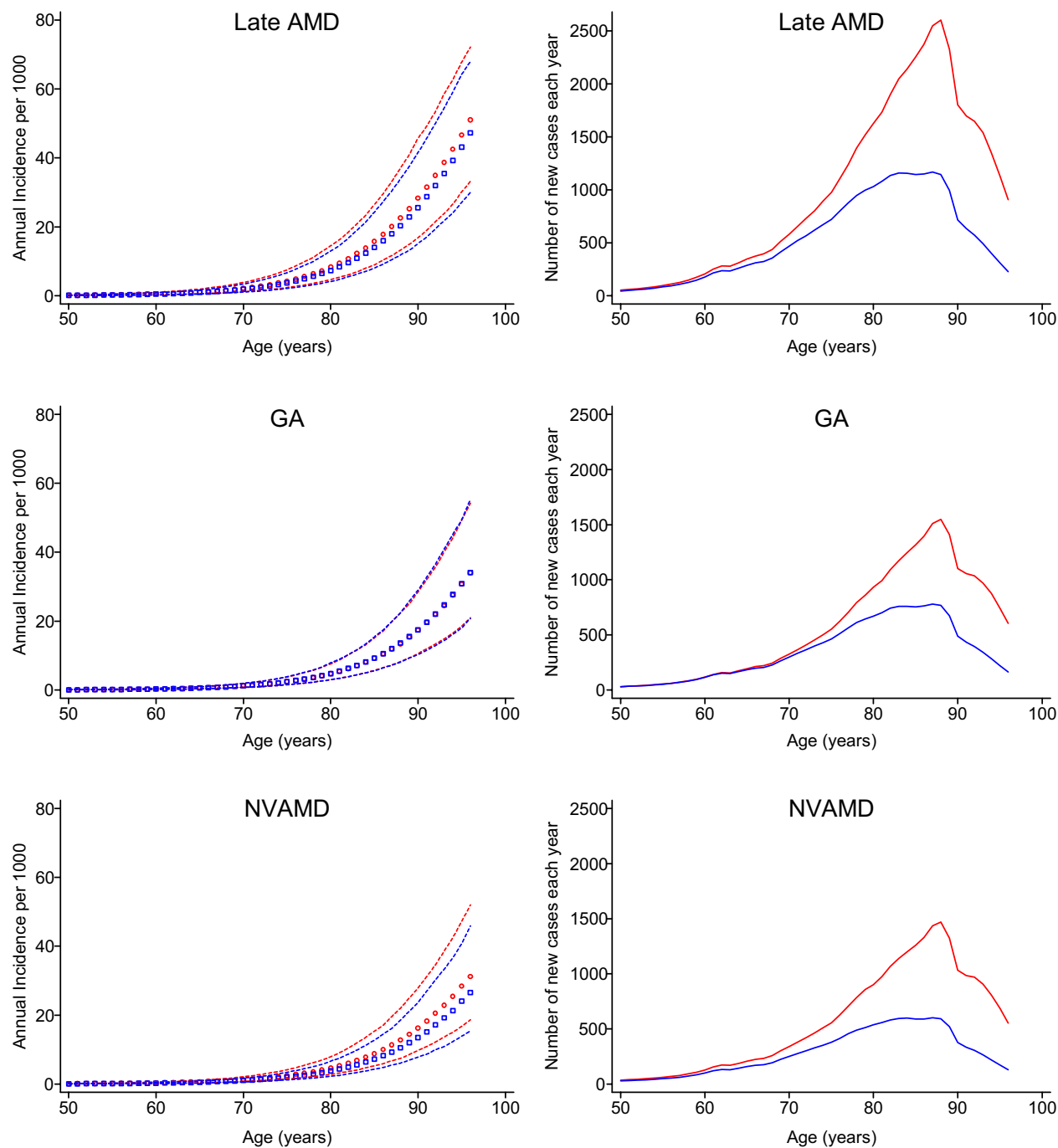
model the number of prevalent cases of late AMD, GA and NVAMD. We estimate that there are currently 513000 (95\% Cr I 363000 to 699000$)$ prevalent cases of late AMD, 276000 cases of GA (95\% CrI 188000 to 396000) and 263000 cases of NVAMD (95\% CrI 185000 to 361000$)$. With knowledge of the probability of death by age, and assuming similar mortality in those with and without AMD, the annual number of incident cases is 71200 (95\% CrI 41100 to 120900), 43700 (95\% CrI 27000 to 71200 ) and 39700 (95\% CrI 23700 to 68300), respectively. While women have slightly higher age-specific prevalence rates of late and NVAMD, it is the much greater number of older women in the UK that results in the gender difference in the number of prevalent and incident cases (figures 1 and 3 ). This explains the perceived view of an abundance of older women presenting to medical retina clinics with $\mathrm{AMD}$.

How do these numbers compare with previous estimates? Numbers from this study are similar to, but more precise than, our earlier estimates of prevalent cases of 191000 (95\% CI 171000 to 310000 ) with GA and 271000 (95\% CI 179000 to 405000 ) with NVAMD, which combine to give 462000 with late AMD within the present study's CrIs. Our CrIs also include recent point estimates ${ }^{3}$ derived by applying findings from another review ${ }^{27}$ to the UK population (although our NVAMD estimates are lower). There have also been a number of attempts to estimate incidence of NVAMD in the UK in order to plan treatment demand and costs. ${ }^{28}$ We are not aware of any UK estimates for GA incidence, probably because this is less of a priority given lack of effective treatment (although the need for low vision services remains). Initial estimates of NVAMD requiring photodynamic therapy in England and Wales suggested 5000 patients per year, estimated from either blind and partial sight registrations ${ }^{10}$ or from two non-UK prospective cohort studies. ${ }^{11} 2930$ With the introduction of newer therapies for NVAMD, ${ }^{5}$ potentially suitable for classic and occult types of choroidal neovascularisation, estimates of the number eligible for treatment have increased from 7000 to 26000 patients per year. ${ }^{28}$ The source of these estimates is unclear, ${ }^{28}$ but Novartis Pharmaceuticals UK also suggested 26000 new cases of wet $\mathrm{AMD}$ in the UK per year, reported as being calculated from our earlier review of prevalence. ${ }^{2} 31$ This figure is commensurate with estimates that there are 13000 to 37000 incident cases of NVAMD in England and Wales per year, ${ }^{28}$ based on two prospective cohort studies in the Netherlands ${ }^{32}$ and Australia. ${ }^{8}$ Our prediction of incidence is higher, but our CrIs are wide and include many of the previously reported estimates (including the popular figure of 26000 annual cases of NVAMD). Our overall and age-specific annual incidence rates (Web table 2) also appear to be higher than those calculated ${ }^{33}$ from 'first eye' cumulative AMD incidence in other studies. ${ }^{6-9}$ The exponential rise in prevalence and incidence rates with age (with prevalence rates of late AMD quadrupling per decade $)^{4}$ makes direct comparisons with estimates from individual studies difficult because they are heavily dependent on the age distribution of the sample at baseline and period of follow-up. Representation of data from older people (especially those aged 80 years or more) is needed to avoid underestimation of population numbers with late stage $\mathrm{AMD}$ (while recognising that the number with or developing $A M D$ in a population will ultimately fall with age due to increased mortality). Unfortunately, any single prospective study has limited information on incidence at older ages when AMD is particularly prevalent. Although there are some studies with long durations of follow-up (up to 15 years), ${ }^{813} 14$ results are generally reported as 5- or 10-year incidence rates making it difficult to determine the annual incidence without knowledge of the change in pattern of incidence with time/age. Therefore, estimating incidence from prevalence, particularly at older ages, is an efficient approach and likely to be more accurate in the absence of large studies with follow-up at all ages (particularly at older ages where follow-up is more problematic).

Our numbers are calculated from either eye prevalence rates. ${ }^{4}$ While this might overestimate any potential visual loss associated with the condition (especially when both eyes are used), this is appropriate when estimating potential therapeutic costs as bilateral disease is less common, and presence of any disease may warrant treatment. Using 'either eye' case definitions will result in some individuals being counted in GA and NVAMD groups, but this number is likely to be small. While this may partly explain why the estimated number with GA and NVAMD combined is higher than those estimated with late $\mathrm{AMD}$, a more plausible explanation is that the estimates of prevalence of late AMD are more precise as they are estimated from a meta-analysis of a larger number of studies (31 studies) compared with those for GA and NVAMD (23 studies). Similar to earlier work estimating incidence of glaucoma from agespecific prevalence, ${ }^{22}$ our calculations assume that: (i) AMD is life-long after diagnosis, (ii) overall mortality risks are the same in those with and without AMD and (iii) AMD is stable in the population (with risk factors for the condition remaining the same). The first assumption is correct, there is no consistent evidence to argue against the second ${ }^{34-36}$ and we have found no strong evidence in our meta-analysis that late $\mathrm{AMD}$ prevalence has changed over time, ${ }^{4}$ although a recent study suggested that the prevalence of AMD (including early and late forms) may have fallen, ${ }^{37}$ attributed to improved diets and reductions in smoking prevalence. ${ }^{38}$ The estimation of incidence for older age groups is the most plausible explanation for our higher numbers, with the peak in number of prevalent and incident cases being just below 90 years of age. The noticeable kink in the decline beyond 90 years of age reflects the sharp decline in births during World War I (1914-1918), followed by the rapid postwar baby boom (approximately 1920). ${ }^{39}$ While our estimates of NVAMD incidence are high, this may not reflect the number in need of treatment. The type of NVAMD on presentation cannot be estimated from these data, that is, classic or occult, although limited evidence from other sources suggests a ratio of $2: 1{ }^{11}$ Moreover, older patients may be too frail to benefit/undergo treatment.

In the absence of any large, geographically representative population study of $\mathrm{AMD}$ in the UK, this review provides the best estimates of the number of prevalent and incident cases of late AMD, GA and NVAMD in the largely white older population of the UK. While most of the current older UK population are white $(96 \%$ white population in England and Wales aged $\geq 65$ years), ${ }^{40}$ this is likely to diminish in the future. However, evidence suggests similar or lower rates of AMD in populations of non-European ancestry. ${ }^{27} 41$ Our estimates are based on pooled findings from studies carried out in white populations from North America, Australia and Europe with shared ancestry (including recent ${ }^{23}$ and historical studies in the $\mathrm{UK}^{24-26}$ ). All studies contributed to the prevalence estimates used to calculate incidence. We adjusted for differences between studies in methodology which may have biased rate estimates ${ }^{24-26}$ (such as no fundus imaging and study-specific as opposed to internationally recognised disease definitions). ${ }^{15} 16$ Projections over the next decade suggest that the number of prevalent cases of late AMD will increase steadily by a third by 2020 due to population ageing. These evidence based estimates can be used to help plan social and healthcare provision for the present and the future. 
Funding The work was supported by a grant from the Macular Diseases Society and commissioned on behalf of the Macular Interest Group of Vision 2020, UK.

Competing interests None.

Contributors All authors contributed to conception and design of this paper. ARR carried out the statistical analysis. CGO and ARR conceived and raised funding for this study with help from RW and AEF. The paper was critically appraised by all authors for intellectual content; CGO drafted the paper and is the guarantor. The guarantor accepts full responsibility for the integrity of the work as a whole. All authors had access to the data and approved the final version to be published.

Provenance and peer review Not commissioned; externally peer reviewed.

\section{REFERENCES}

1. Bunce C, Xing W, Wormald R. Causes of blind and partial sight certifications in England and Wales: April 2007-March 2008. Eye (Lond) 2010;24:1692-9.

2. Owen CG, Fletcher $A E$, Donoghue $M$, et al. How big is the burden of visual loss caused by age related macular degeneration in the United Kingdom? Br J Ophthalmol 2003;87:312-17.

3. Minassian DC, Reidy A, Lightstone A, et al. Modelling the prevalence of age-related macular degeneration (2010-2020) in the UK: expected impact of anti-vascular endothelial growth factor (VEGF) therapy. Br J Ophthalmol 2011;95:1433-6.

4. Rudnicka AR, Jarrar Z, Wormald R, et al. Age and gender vairations in age realted macular degeneration prevalence in populations of European ancestry: a meta-analysis. Ophthalmology. Published Online First: 2011. doi:10.1016/..ophtha.2011.08.038

5. Wong TY, Liew G, Mitchell P. Clinical update: new treatments for age-related macular degeneration. Lancet 2007;370:204-6.

6. van Leeuwen R, Klaver CC, Vingerling JR, et al. Epidemiology of age-related maculopathy: a review. Eur J Epidemiol 2003;18:845-54.

7. Buch H, Nielsen NV, Vinding T, et al. 14-year incidence, progression, and visual morbidity of age-related maculopathy-The Copenhagen City Eye Study. Ophthalmology 2005;112:787-98.

8. Mitchell P, Wang JJ, Foran S, et al. Five-year incidence of age-related maculopathy lesions: the Blue Mountains Eye Study. Ophthalmology 2002;109:1092-7.

9. Klein R, Klein BE, Tomany SC, et al. Ten-year incidence and progression of age-related maculopathy. Ophthalmology 2002:109:1767-79.

10. Harding S. Photodynamic therapy in the treatment of subfoveal choroidal neovascularisation. Eye (Lond) 2001;15:407-12

11. Meads C, Salas C, Roberts T, et al. Clinical effectiveness and cost-utility of photodynamic therapy for wet age-related macular degeneration: a systematic review and economic evaluation. Health Technol Assess 2003;7:v-98.

12. The Royal College of Ophthalmologists. Commissioning Contemporary AMD Services: A Guide for Commissioners and Clinicians. 2007. http://www.rcophth.ac.uk/ documents.asp?section=39\&sectionTitle=Publications (accessed Jan 2010)

13. Klein R, Klein BE, Knudtson MD, et al. Fifteen-year cumulative incidence of age-related macular degeneration. Ophthalmology 2007;114:253-62.

14. Wang JJ, Rochtchina E, Lee AJ, et al. Ten-year incidence and progression of age-related maculopathy-The Blue Mountains Eye Study. Ophthalmology 2007:114:92-8.

15. Bird AC, Bressler NM, Bressler SB, et al. An international classification and grading system for age-related maculopathy and age-related macular degeneration. The International ARM Epidemiological Study Group. Surv Ophthalmol 1995:39:367-74.

16. Klein R, Davis MD, Magli YL, et al. The Wisconsin age-related maculopathy grading system. Ophthalmology 1991;98:1128-34.

17. Office for National Statistics. Population Estimates for UK, England and Wales, Scotland and Northern Ireland, 2007. http://www.ons.gov.uk/ons/rel/pop-estimate/ population-estimates-for-uk-england-and-wales-scotland-and-northern-ireland/ 2007/index.html (accessed Sep 2011).

18. Office for National Statistics. Population Estimates for UK, England and Wales, Scotland and Northern Ireland, 2008. 2009. http://www.ons.gov.uk/ons/rel/popestimate/population-estimates-for-uk-england-and-wales-scotland-and-northernireland/2008/index.html (accessed Sep 2011).
19. Office for National Statistics. Population estimates for UK, England and Wales, Scotland and Northern Ireland, 2009. 2009. http://www.ons.gov.uk/ons/rel/popestimate/population-estimates-for-uk-england-and-wales-scotland-and-northernireland/2009/index.html (accessed Sep 2011).

20. Office for National Statistics. United Kingdom, Interim Life Tables, 1980-82 to 2007-09. 2010. http://www.ons.gov.uk/ons/publications/re-reference-tables.html? edition $=$ tcm\%3A77-61850 (accessed Sep 2011)

21. Office for National Statistics. UK Population Estimates and Projections 19712083. 2010. http://www.ons.gov.uk/ons/taxonomy/index.html?nscl=Population +Estimates + by+Age+and +Sex (accessed Sep 2011).

22. Leske MC, Ederer F, Podgor M. Estimating incidence from age-specific prevalence in glaucoma. Am J Epidemiol 1981;113:606-13.

23. Augood CA, Vingerling JR, de Jong PT, et al. Prevalence of age-related maculopathy in older Europeans: the European Eye Study (EUREYE). Arch Ophthalmol 2006:124:529-35.

24. Das BN, Thompson JR, Patel R, et al. The prevalence of eye disease in Leicester-a comparison of adults of Asian and European descent. J R Soc Med 1994:87:219-22.

25. Gibson JM, Rosenthal AR, Lavery J. A study of the prevalence of eye disease in the elderly in an English community. Trans Ophthalmol Soc U K 1985; 104:196-203.

26. Pauleikhoff $\mathbf{D}$, Wormald RP, Wright $\mathrm{L}$, et al. Macular disease in an elderly population. German J Ophthalmol 1992;1:12-15.

27. Friedman DS, O'Colmain BJ, Munoz B, et al. Prevalence of age-related macular degeneration in the United States. Arch Ophthalmol 2004;122:564-72.

28. Colquitt JL, Jones J, Tan SC, et al. Ranibizumab and pegaptanib for the treatment of age-related macular degeneration: a systematic review and economic evaluation. Health Technol Assess 2008:12:iii-iv, ix-201.

29. Klein R, Klein BEK, Jensen SC, et al. The 5-year incidence of age-related maculopathy in the Beaver Dam Eye Study. Invest Ophthalmol Vis Sci 1996;37:1916

30. Klaver CC. Assink JJ, Van LR, et al. Incidence and progression rates of age-related maculopathy: the Rotterdam Study. Invest Ophthalmol Vis Sci 2001:42:2237-41.

31. National Institute for Health and Clinical Excellence. Ranibizumab and Pegaptanip for the Treatment of Age-related Macular Degeneration: Response to Consultee and Commentator Comments on the draft scope. 2006. http://www.nice. org.uk/nicemedia/live/11700/34997/34997.pdf (accessed Jun 2011).

32. Van Leeuwen R, Klaver CC, Vingerling JR, et al. The risk and natural course of agerelated maculopathy: follow-up at $61 / 2$ years in the Rotterdam study. [Erratum appears in Arch Ophthalmol. 2003 Jul;121(7):955]. Arch Ophthalmol 2003;121:519-26.

33. Karnon J, Czoski-Murray C, Smith K, et al. A preliminary model-based assessment of the cost-utility of a screening programme for early age-related macular degeneration. Health Technol Assess 2008;12:iii-iv, ix-124.

34. Cugati S, Cumming RG, Smith W, et al. Visual impairment, age-related macular degeneration, cataract, and long-term mortality: the Blue Mountains Eye Study. Arch Ophthalmol 2007;125:917-24.

35. Thiagarajan M, Evans JR, Smeeth L, et al. Cause-specific visual impairment and mortality: results from a population-based study of older people in the United Kingdom. Arch Ophthalmol 2005;123:1397-403.

36. Xu L, Wang YX, Wang J, et al. Mortality and ocular diseases: the Beijing Eye Study. Ophthalmology 2009;116:732-8.

37. Chakravarthy U, Wong TY, Fletcher A, et al. Clinical risk factors for age-related macular degeneration: a systematic review and meta-analysis. BMC Ophthalmol 2010;10:31.

38. Thornton J, Edwards R, Mitchell P, et al. Smoking and age-related macular degeneration: a review of association. Eye (Lond) 2005:19:935-44.

39. Hicks J, Allen G. A Century of change: Trends in UK Statistics since 1900. Res paper RP99/111. 1999 http://www.parliament.uk/briefing-papers/RP99-111 (accessed Jun 2011).

40. Office for National Statistics. Current Estimates-Population Estimates by Ethnic group Mid-2009. 2011. http://www.statistics.gov.uk/statbase/product.asp? vlnk=14238 (accessed Jun 2011).

41. Kawasaki R, Yasuda M, Song SJ, et al. The prevalence of age-related macular degeneration in Asians: a systematic review and meta-analysis. Ophthalmology 2010;117:921-7 